UAHEP053

\title{
Properties of a future susy universe
}

\author{
L. Clavelli* \\ Department of Physics and Astronomy \\ University of Alabama \\ Tuscaloosa AL 35487
}

October, 2005

\begin{abstract}
In the string landscape picture, the effective potential is characterized by an enormous number of local minima of which only a minuscule fraction are suitable for the evolution of life. In this "multiverse", random transitions are continually made between the various minima with the most likely transitions being to minima of lower vacuum energy. The inflationary era in the very early universe ended with such a transition to our current phase which is described by a broken supersymmetry and a small, positive vacuum energy. However, it is likely that an exactly supersymmetric (susy) phase of zero vacuum energy as in the original superstring theory also exists and that, at some time in the future, there will be a transition to this susy world. In this article we make some preliminary estimates of the consequences of such a transition.
\end{abstract}

PACS numbers: 11.30.Pb, 12.60.J, 13.85.-t

\section{Introduction}

Current indications are that the universe began about $10^{10} \mathrm{bc}$ in a phase of large vacuum energy density resulting in a rapid expansion. In the very early instants the universe underwent a transition to the current phase characterized by a vacuum energy density

$$
\epsilon=(0.0023 \quad e V)^{4}
$$

*lclavell@bama.ua.edu 
small enough to allow the evolution of stars, planets, and life. Although this vacuum energy is more than 100 orders of magnitude below the "natural" scale of $M_{P l}^{4}$ it is argued that its existence is made likely by the sheer number of minima in the string landscape [1]. A possible artist's conception of a piece of the string landscape is given in figure 1

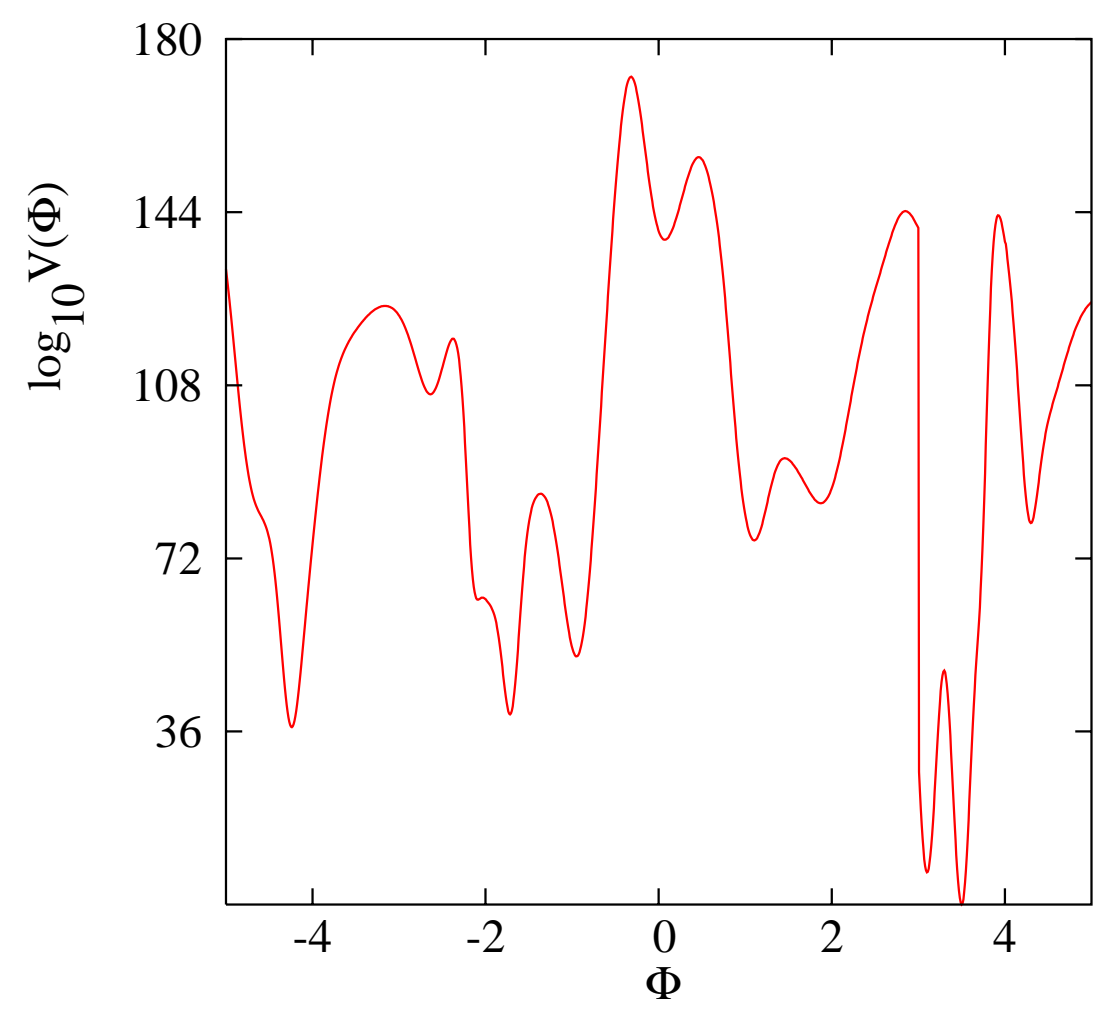

Figure 1: A schematic representation of the effective potential in the string landscape picture. The potential is measured in units of $\mathrm{MeV} / \mathrm{m}^{3}$ and the $\mathrm{y}$ axis has a broken scale taken to be linear in $\mathrm{V}$ at low values of the potential. The low lying valleys have a bi-cuspid nature characterized by a broken susy as well as an exact susy minimum.

The string landscape shows promise 2] for the understanding of several features of the inflationary era and its subsequent transition to the current phase. In order to provide a long enough period of inflationary growth, it is currently assumed that the transition took place via a "slow roll" rather than a sudden quantum jump. Perhaps, the slow roll was actually a long sequence of jumps between progressively lower minima. Although the details remain problematic, it is clear that such a transition out of an inflationary era was a prerequisite for the evolution of life.

It is widely believed that the current phase of the universe is one of broken supersymmetry where the partners of the standard model particles are in the hundred $\mathrm{GeV}$ mass region or above. In the Cern theory group this universe has been referred to as "Susonia". One of the primary goals of the Large Hadron Collider presently under construction at Cern is 
to establish that this is indeed our universe. Just as the existence of water in one phase can prompt a search for other phases of water, confirmation of the existence of Susonia would give added impetus to the search for other phases of supersymmetry. Since Quantum Chromodynamics seems almost certain to exist in at least two phases, it should not be surprising if more than one phase of supersymmetry exists.

It is very likely that, in addition to our universe with a vacuum energy given by eq. 1.1. there exists a lower minimum of the effective potential corresponding to an exact supersymmetry. This seems to be a persistent prediction of superstring theory. In flat space such a universe would have vanishing vacuum energy. It is possible that other minima of negative vacuum energy corresponding to anti-deSitter phases also exist. If these minima are supersymmetric and have a vacuum energy that is not too large in absolute value, many of our considerations would remain valid although such a universe would ultimately collapse toward a big crunch. For definiteness we consider primarily the phase of zero vacuum energy as the true vacuum. Figure 2 illustrates the double well potential in which the false vacuum is our broken susy universe and the true or absolute vacuum is the world of exact susy.

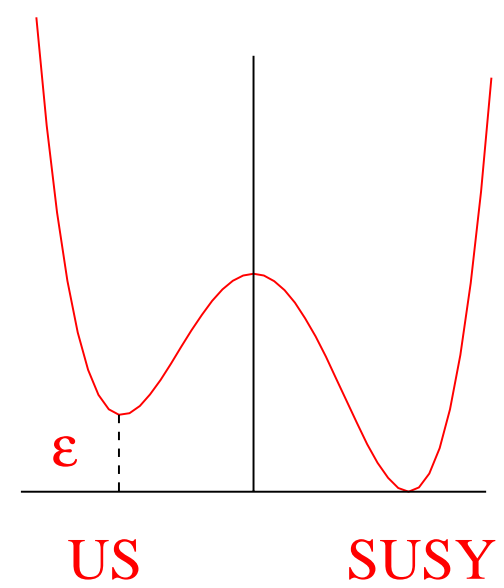

Figure 2: The double well effective potential representing our broken susy world and a nearby world of exact susy.

In the string landscape picture, such a world could exist now beyond our visible universe or in localized bubbles within it. In any case, given a supersymmetric absolute minimum of vacuum energy less that that of eq. 1.1 and assuming the effective potential is dynamically determined, there will inevitably be a future transition from our broken susy world to a world of exact susy. This world of perfect susy might well be the final phase of our universe. Either of these considerations could justify some theoretical exploration of these alternate worlds. An initial study in string theory of the transition from a deSitter world of positive vacuum energy to an exact susy ground state has already been made [3].

The world of broken supersymmetry is dominated by the Pauli principle. Every atom above Helium is characterized by energy permanently stored in a Pauli tower of electrons and 
in a separate tower of nucleons in the atomic nucleus. In exact susy, conversion of fermion pairs to degenerate scalar pairs not governed by the Pauli principle allows the release of this energy.

\section{Susyria and Susalia}

The physics of bulk supersymmetric matter is very much terra incognita. Many questions cannot be easily answered. Only the simplest issues are addressed here. We tentatively assume that the primary differences between our world and the world of exact susy are the degeneracy of the susy multiplets and the diminished importance of the Pauli principle.

For definiteness, we explore a world governed by the minimal supersymmetric standard model (MSSM) with all the susy breaking parameters set to zero. We name it "Susyria". In this world each particle-sparticle pair has a common mass equal to that of the standard model particle in the broken susy phase with coupling strengths approximately as in broken susy. Clearly, other nearby susy worlds [4 are equally deserving of study as are broken susy deSitter and anti-deSitter worlds of vacuum energy less in absolute value than that of our world. String theory, however, while presenting some general possibilities, has few specific predictions at the present time. The question of the dimensionality and topology of space time in a future phase has also received some attention [5]. In view of the attractive notion of radiative breaking of the electroweak symmetry, another high priority goal would be the world of exact susy and also exact electroweak gauge invariance. In such a totally symmetric world, "Susalia", all fermions would be massless. However, at present, this world is beyond reach since we have no calculational techniques for studying the interactions of massless charged particles. Perhaps in Susalia the gauge group is left-right symmetric to allow for particle masses.

In cosmology one must distrust arguments based on "naturalness". The low vacuum energy of our world given by eq. 1.1 must at present be considered very unnatural although our existence depends on it. In fact, if a theorist in some other world were presented with the standard model Lagrangian with its nineteen free parameters, he would conclude that the evolution of life in such a universe would be highly unlikely since the necessary parameters would probably form a set of measure zero.

For example, in our world of broken symmetry, the neutron is heavier than the proton with no present explanation other than an anthropic coincidence among the Higgs yukawa couplings. We use the word "coincidence" to describe a fact for which there is as yet no physics explanation in the traditional sense without implying that none will ever be found. The coincidence among quark masses is illustrated in fig. 3

As a function of generation number, the up quark mass curve is slightly concave downward on a log plot while the down-type quark mass curve is slightly concave upward. The pattern of baryon masses follows the pattern of quark masses. If the neutron were $1 \%$ heavier, other things being equal, the only stable atom would be Hydrogen since the nuclei of heavier atoms would $\beta_{-}$decay followed sometimes by proton ejection. If the neutron were $1 \%$ lighter 


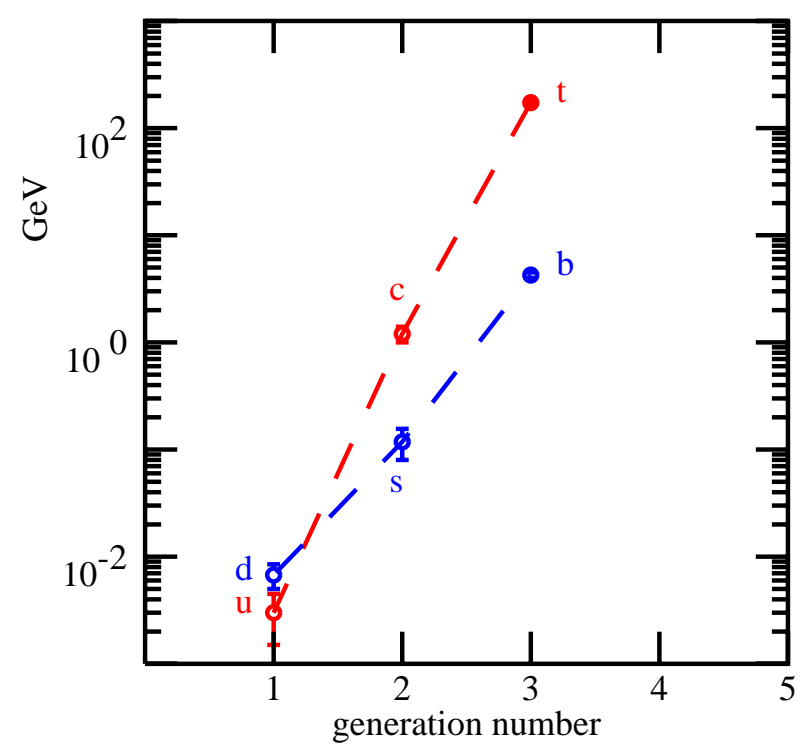

Figure 3: The up-type and down-type quark masses as a function of generation. Quark masses are taken from the Particle Data Group review [6]. In the two heavier families the up-type quark is heavier than the down-type quark. This is reversed in the first generation.

all protons would $\beta_{+}$decay followed sometimes by neutron ejection and there would be no atoms. In either case no presently conceivable life forms could exist. In this sense our life depends on the unexplained smallness and relative sign of the quadratic terms in the mass formulae.

Some questions we would like to answer are

1. Could life have arisen if there had been a phase transition directly from the inflationary era to the exact susy minimum? If not, there might be an anthropic lower limit to the vacuum energy density in addition to Weinberg's anthropic upper limit [8].

2. Could life survive, or re-establish itself, following a transition from our broken susy world to the exact susy world? If the answer to the first question is "no" and the answer to the second question is "yes" there could be an anthropic understanding, after the transition, of why the universe existed for a time in the broken susy phase.

3. What would be the primary characteristics of the physics (and biology, if any,) of the exactly supersymmetric phase?

4. Can we estimate the probable time remaining before our universe converts to a susy world? 
The primary goal of this preliminary theoretical expedition is, perhaps, the search for life in other worlds. As alluded to above, the absence of life in other universes might help explain why our present universe is as it is. However, a priori, it cannot be ruled out that Susyria is a new Galapagos teeming with abundant life. Therefore, when ambiguities present themselves, we will tentatively assume the solution seemingly most favorable to life. These choices are motivated by a desire to determine if there are any reasonable possibilities that are consistent with the evolution of susy life and, obviously, no claim is made that no other possibilities are present.

In Susyria many of the anthropic coincidences of our world would continue to hold. The neutron proton mass difference discussed above would be, at least roughly, as in our world. Similarly, the electromagnetic and strong coupling constants would be approximately as in our world. Slight differences in masses and coupling strengths might be found by requiring grand unification and extrapolating to low energies in exact supersymmetry. Such arguments would suggest that each of the gauge coupling constants at low energies are somewhat smaller in an exact susy universe than in our broken susy world. To see this it is sufficient to compare the one loop running of the couplings. The two loop results do not significantly change the answers and could be incorporated as in [7. With susy broken at energy scale, $M_{S}$, the three couplings at the $Z$ are

$$
\alpha_{i}\left(M_{Z}\right)^{-1}=\alpha_{0}^{-1}+\left(b_{i}-b_{i}^{S}\right) \ln \left(M_{S} / M_{Z}\right)+b_{i}^{S} \ln \left(M_{G} / M_{Z}\right)
$$

where the differences between the susy and standard model beta function coefficients are

$$
\begin{aligned}
& b_{1}^{S}-b_{1}=\frac{1}{2 \pi}\left(2 N_{f} / 3+N_{H} / 5\right) \\
& b_{2}^{S}-b_{2}=\frac{1}{2 \pi}\left(4 / 3+2 N_{f} / 3+N_{H} / 3\right) \\
& b_{3}^{S}-b_{3}=\quad \frac{1}{2 \pi}\left(2+2 N_{f} / 3\right) .
\end{aligned}
$$

The number of Higgs and fermion families are nominally $N_{H}=1$ and $N_{f}=3$. In exact susy the central term in eqs.2.1 is absent. Thus, assuming the GUT-scale parameters are not affected by the low energy breaking or non-breaking of susy, the relation between the couplings at the $Z$ in an exact susy universe and the couplings in our broken world is

$$
\left(\alpha_{i}^{S}\right)^{-1}\left(M_{Z}\right)-\alpha_{i}^{-1}\left(M_{Z}\right)=\left(b_{i}^{S}-b_{i}\right) \ln \left(M_{S} / M_{Z}\right)
$$

Since the three beta function differences of eqs.2.3 are positive definite, it would be expected that the couplings in an exact susy world are slightly less than those in our universe. extrapolating from the $Z$ to low energy the active number of fermions decreases stepwise. Roughly speaking, therefore, we would expect that the susy fine structure constant would be

$$
\left(\alpha^{S}\right)^{-1} \approx \alpha^{-1}+\frac{1}{3 \pi} \ln \left(M_{S} / M_{e}\right) \approx 138.5
$$

Similarly, the difference between the strong coupling constants at $1 \mathrm{GeV}$ (and therefore perhaps the difference between the $\pi$-Nucleon couplings) would be governed approximately by

$$
\left(\alpha_{s}^{S}(1 G e V)\right)^{-1} \approx \alpha_{s}(1 G e V)^{-1}+\frac{1}{\pi} \ln M_{S} /(1 G e V) \approx 10 .
$$


Susalia, the susy island universe where electroweak breaking is also absent, would also be quite interesting to explore if the problem of massless charged particles could be solved. However, in such a world, one might expect heavier protons than neutrons which might preclude the existence of planets and life.

\section{The nuclear mass formula}

In Susonia, the broken susy world, the masses of nuclei are given, generally to better than $0.1 \%$, by the semi-empirical formula [9]

$$
M(Z, A)=N M_{n}+Z M_{p}-a_{v} A+a_{s} A^{2 / 3}+a_{c} \frac{Z^{2}}{A^{1 / 3}}+a_{a} \frac{(Z-N)^{2}}{A^{2 / 3}}+\frac{\delta}{A^{1 / 2}}
$$

where

$$
\begin{aligned}
a_{v} & =15.67 \mathrm{MeV} \\
a_{s} & =17.23 \mathrm{MeV} \\
a_{c} & =0.714 \mathrm{MeV} \\
a_{a} & =93.15 \mathrm{MeV} \\
\delta & =-11.5 \mathrm{MeV}(\text { even }- \text { even }) \\
& =0(\text { even }- \text { odd }) \\
& =11.5 \mathrm{MeV}(\text { odd }- \text { odd })
\end{aligned}
$$

In addition to the volume term, $a_{v}$, surface term, $a_{s}$, and the Coulomb repulsion term, $a_{c}$, there is the asymmetry term, $a_{s}$, and the pairing term, $\delta$. These last two are direct consequences of the Pauli exclusion principle among fermionic constituents. In the susy phase, the Pauli principle can be evaded by pair conversion from fermions to bosons allowing all particles to drop into ground state energy levels.

$$
f f \rightarrow \tilde{f} \tilde{f}
$$

The added energy required to store fermions would then be eliminated. We would therefore expect nuclear masses in the susy phase to be governed by a formula of the form

$$
M_{s}(Z, A)=N M_{n}+Z M_{p}-a_{v} A+a_{s} A^{2 / 3}+a_{c} \frac{Z^{2}}{A^{1 / 3}}-\frac{11.5 M e V}{A^{1 / 2}} .
$$

Note that, although the final state is a sfermion pair and not a sfermion-antisfermion pair, this process occurs in supersymmetry without $\mathrm{R}$ parity violation. The argument from grand unification 2.42.5 suggests only a few percent difference between coupling constants in the broken susy and exact susy universes. It is, therefore, not unreasonable to assume that couplings in Susyria have the same strengths as in the broken susy phase so, as a zeroth order approximation, we can use the numerical values from eq.2.7. Of course, in addition to the strength of the nuclear force, the average binding energy per nucleon governed by coefficients $a_{v}$ and $a_{s}$ could also be affected by the necessity to put fermions in higher (less tightly 
bound) states. This could lead to somewhat higher average binding energies per nucleon in a supersymmetric nucleus. There will be two types of fermionic protons, one having zero squarks and one having two squarks. The same holds for fermionic neutrons. Stable susy nuclei will have no more than two fermionic protons of each type and no more than two fermionic neutrons of each type with the remaining nuclear charge and atomic number filled out by sprotons and sneutrons. Similarly, the lepton clouds surrounding nuclei would have at most two electrons with the remaining leptonic charge being carried by selectrons. Ground state atomic and nuclear orbitals would be s-wave only although excited states of higher angular momentum would exist. Thus, magnetic moments in Susyria are much weaker than in Susonia. Since, in Susonia, p-wave orbitals are prominent in the widespread phenomenon of double covalent binding, molecular and condensed matter physics would be quite different in Susyria where there might be no pronounced shell structure. Are all the elements of Susyria, therefore, inert like the Helium of Susonia? Or do, at least, the atoms with a single fermionic electron bind as does Susonic Hydrogen? Alternatively, does a large cloud of s-wave selectrons make it energetically favorable for one or more to be bumped up into p-wave states or even for s wave electrons to be more easily shared? These questions cannot be answered without detailed atomic physics calculations. Following our stated program of investigating assumptions that might allow the possibility of life until proven untenable, we will tentatively assume that molecular binding does occur in a susy world. Otherwise we have our answer to questions 1 and 2 of the introduction and Susyria is a totally sterile world.

Although supersymmetric quantum mechanics has proven useful as a technicque to deal with normal atomic physics (as for example in ref. [10]), we know of no attempts to predict the atomic properties of atoms in a true supersymmetric background. Similarly, although susy Yang-Mills (YSM) has been extensively investigated, often this is in a zero flavor mode, so that little insight is available for the behavior of susy nuclear levels. Given the difficulty of explaining nuclear binding from fundamental Quantum Chromodynamics, it is not surprising that SYM is in a very early stage of providing an understanding of susy nuclear physics.

A susy nucleus (snucleus) would undergo $\beta_{-}$decay if

$$
M_{s}(Z, A)-M_{s}(Z+1, A)-m_{e}>0
$$

or $\beta_{+}$decay if

$$
M_{s}(Z, A)-M_{s}(Z-1, A)-m_{e}>0 .
$$

In the standard model, eq. 2.6. the stable nuclei have atomic weights greater than but of the same order of magnitude as their atomic numbers. Using eq. 2.9, one can see that the stable snuclei as a function of atomic number, $Z$, would have very large atomic weights compared to the same elements in the broken susy world.

$$
A_{\min }(Z)<A<A_{\max }(Z)
$$

with

$$
A_{\text {min }}(Z)=\left(\frac{2 a_{c}(Z-1 / 2)}{M_{n}-M_{p}+m_{e}}\right)^{3}
$$


and

$$
A_{\text {max }}(Z)=\left(\frac{2 a_{c}(Z+1 / 2)}{M_{n}-M_{p}-m_{e}}\right)^{3} .
$$

Using the Coulomb coefficient, $a_{c}$, from eq. 2.7 the low-lying stable susy nuclei are as given in table 1. Coulomb repulsion disfavors a large number of protons or sprotons, the Pauli principle disfavors a large number of neutrons, but there is no principle disfavoring large numbers of sneutrons.

\begin{tabular}{||lcc||}
\hline Hydrogen & $Z=1$ & $1<A<19$ \\
Helium & $Z=2$ & $3<A<88$ \\
Lithium & $Z=3$ & $8<A<243$ \\
Berylium & $Z=4$ & $21<A<518$ \\
Boron & $Z=5$ & $45<A<946$ \\
Carbon & $Z=6$ & $82<A<1562$ \\
Nitrogen & $Z=7$ & $136<A<2400$ \\
Oxygen & $Z=8$ & $209<A<3494$ \\
Fluorine & $Z=9$ & $304<A<4878$ \\
& & \\
\hline
\end{tabular}

Table 1: Atomic weights of the stable isotopes of low-lying elements in the exact susy limit of the MSSM. Elements up to $\mathrm{He}^{4}$ would have the same masses as in the standard model.

\section{fission and fusion}

In the standard model, nuclear binding energies per nucleon, as described by eq. 2.6. increase up to Iron and then decline. The result is that Iron is the end point of nuclear fusion. The decline in binding energies above Iron allows very heavy elements to approach the point of spontaneous fission. It is estimated that, for spontaneous fission to occur, one must have [1]

$$
\frac{Z^{2}}{A}>44
$$

This ratio is proportional to the ratio of coulomb energy to surface tension in a heavy nucleus. In the broken susy world this condition is never attained although, for a few elements, notably Uranium and Thorium, it is sufficiently closely approached that slow neutrons can induce fission.

On the other hand, in the susy world being explored here, the nuclear binding energies defined by eq. 2.9 increase monotonically. The result is that stellar lifetimes may be greatly prolonged since fusion will not end at iron. For example, the energy release from alpha induced fusion 


$$
\alpha+(Z, A) \rightarrow(Z+2, A+4)
$$

is predicted from eq. 2.9 to be roughly $30 \mathrm{MeV}$ nearly independent of $\mathrm{A}$. The reaction 2.16] would be followed by beta decay processes down to stable nuclei consistent with eq. 2.12

Fusion of Susy Hydrogen and Helium proceeds at a similar rate as in the broken susy world except for the statistical effect of the large number of stable isotopes of the low lying elements. Whether these effects would be sufficient to preclude the evolution of stars, planets, and life if the susy phase transition had occured in the very early universe is still uncertain.

It is deducible from the form of eq. 2.9 that there are no exothermic fission reactions and no alpha decays in Susyria. If we extend table 1 to include a few heavier elements that are common or problematic in Susonia, it is clear that their extreme atomic weights probably preclude their existence in a susy world except, perhaps, in extremely small trace concentrations.

\begin{tabular}{||lcc||}
\hline Silicon & $Z=14$ & $1218<A<17346$ \\
Iron & $Z=26$ & $8209<A<105888$ \\
Arsenic & $Z=33$ & $16994<A<213917$ \\
Lead & $Z=82$ & $267992<A<3195023$ \\
Plutonium & $Z=94$ & $404654<A<4801840$ \\
& & \\
\hline
\end{tabular}

Table 2: Atomic weights of the stable isotopes of a few highly charged nuclei in the exact susy limit of the MSSM. The very large sneutron numbers required to stabilize such nuclei suggest that these elements are nonexistent or extremely rare in a susy universe.

Of course, the seemingly extreme atomic weights of tables 1, 2] could be moderated by future analyses along the lines suggested above. If for example, in exact susy, the fine structure constant were $10 \%$ to $20 \%$ lower than in broken susy or the neutron-proton mass difference were greater by a similar percentage, the large atomic weights of table[ would be significantly reduced without negating our qualitative expectation that susy nuclei would be much heavier, for fixed atomic number, than nuclei in the broken susy world.

\section{Susy Biology}

Is a supersymmetric world capable of sustaining life? We cannot answer this question definitively as yet. However, in our broken susy world, stable elements of atomic weight up to 238 exist. If the transition to exact susy were to take place in an already evolved broken susy world such as ours, all of the elements with atomic weights above 209 would beta decay to Oxygen. Similarly, referring to table 1 , elements with atomic weights between 136 and 208 
would decay to Nitrogen, etc. In this case no elements of atomic number greater than Oxygen would exist unless fusion were ignited. On the other hand, some elements beyond Oxygen could also be produced in this analysis if the Coulomb coefficent, $a_{c}$, in the susy world were somewhat smaller than given in eq. 2.7. The argument from grand unification given above would predict a weaker low energy electromagnetic interaction but not sufficiently weaker to greatly modify the atomic weights in table 1. Other possible effects might need to be investigated.

\begin{tabular}{||l|c||}
\hline & \\
Adenine & $\mathrm{C}_{5} \mathrm{H}_{5} \mathrm{~N}_{6}$ \\
Thymine & $\mathrm{C}_{5} \mathrm{H}_{6} \mathrm{~N}_{2} \mathrm{O}_{2}$ \\
Cytosine & $\mathrm{C}_{4} \mathrm{H}_{5} \mathrm{~N}_{3} \mathrm{O}$ \\
Guanine & $\mathrm{C}_{5} \mathrm{H}_{5} \mathrm{~N}_{5} \mathrm{O}$ \\
& \\
\hline
\end{tabular}

Table 3: The chemical formulae for the four bases that encode all life forms in our universe.

It is interesting that the elements up to Oxygen are sufficient to define the structure of DNA and, therefore, the genetic code of every life form as indicated in table 3

Since these elements also exist in a susy world, each individual of every species has a potential supersymmetric counterpart. The composition of the four basic elements in the human body is summarized in table 4 .

Some 21 elements of atomic number greater than that of Oxygen, though occuring only in minor or trace amounts, play important roles in life. It is not yet clear whether molecular physics allows the four bases to bind and whether the trace amounts of higher elements found in life forms on earth can be dispensed with or replaced by lower elements. The absence of a prominent shell structure suggests a possible affirmative answer to the latter question. We will tentatively assume the answer to both these questions is affirmative.

\begin{tabular}{||l|c||}
\hline \hline & \% by weight \\
\hline Hydrogen & 9.5 \\
Carbon & 18.5 \\
Nitrogen & 3.2 \\
Oxygen & 65.0 \\
\hline Total & 96.2 \\
& \\
\hline \hline
\end{tabular}

Table 4: Composition of the four basic elements in the human body by weight.

In Susyria there are three atoms of each atomic number above Hydrogen having either zero, one, or two fermionic electrons with the remaining charge in the atomic cloud being made up of selectrons. Thus, even if no elements above Oxygen exist, there might be a surprising variety of different molecules. If nuclear differences are relevant, one might note 
that the 148 stable isotopes of susy Carbon are far more numerous than the two stable isotopes of Carbon in the broken susy world. In the nucleus of susy Carbon, there are either zero, one, or two fermionic protons and zero, one, or two fermionic neutrons. Thus there are 1332 different stable species of Carbon nucleus in the susy world. In addition, each proton and each neutron can have either zero, one, two, or three fermionic quarks. Scalar nucleons are those with zero or two fermionic quarks. Since molecular vibrational and rotational frequencies are inversely proportional to the square root of the atomic weights, molecular emission and absorption lines from a susy world would appear strongly red-shifted.

\section{The coming transition}

We have not found a compelling proof that a direct transition from the inflationary universe to a susy universe was impossible from the point of view of allowing for human evolution. An example of such a proof could conceivably be a demonstration that the energy release in supernovae comes from a susy phase transition in dense matter such as proposed for gamma ray bursts [12]. Since supernovae are responsible for seeding the universe with the elements beyond Lithium which are necessary for life, if supernovae could be proven to require a broken susy ground state, it would follow anthropically that we live, at least temporarily, in a broken susy world. It is well known [13] that standard model monte carlos do not succeed in generating an adequate supernova explosion although work in this direction is ongoing. Alternatively, we could note that a fine tuning in the strong force governing the nuclear excitation energy in Carbon was essential in the stellar nucleosynthesis of Carbon and higher elements in the early universe (the triple alpha process) 14, 15. Since this anthropic coincidence would be expected to be de-tuned in an exact susy world due to small differences in the strong coupling constant, it might not be possible to generate elements above Carbon by stellar nucleosynthesis in such a world even if there is a suitable supernova mechanism to distribute them.

Experimentally, in any case, such a direct transition from an inflating state of high vacuum energy to an exact susy world did not take place since we find ourselves in an intermediate broken susy world. Therefore, the final transition must be from our broken susy world with positive vacuum energy to the exact susy ground state.

In the standard astrophysics it is common to assume that the universe will end in an infinitely dilute cold system where $99 \%$ of the mass is in dark dwarfs and the rest being in neutron stars and black holes. If the susy phase transition were to occur at this stage, there might be a multitude of gamma ray bursts but, perhaps, no release of baryonic matter that could lead to a rebirth of the universe in a supersymmetric form. Although such a late stage susy transition might be the most likely, we will also entertain the prospect that the susy transition happens at a time when burning stars are still encircled by planets possibly supporting intelligent life forms.

The final transition will then begin with the quantum nucleation of a susy bubble of critical size which will expand with the speed of light. Inside the growing bubble there will be a new physics and a new astronomy based on the Lagrangian of exact susy. It will 
strike each planet without warning and wash around the globe at the speed of light and through it with, perhaps, the speed of sound. In its wake all the Pauli towers will collapse releasing many times the lethal dose of radiation. For example the nuclear excitation energy released in a human body would be about $10^{15}$ rem compared to the lethal dose of 400 to 450 rem. No known life form, including the resilient cockroach, could survive a radiation burst of this magnitude. Afterwards all the elements will beta decay down to (roughly) susy oxygen and lower elements. It is possible that fusion ignition will afterwards re-balance chemical compositions and produce some elements above Oxygen. However, since there is no activation of long lived radioactive isotopes in a susy world, the radiation will rapidly die out leaving a calm isotopically rich soup of susy elements up to oxygen but not much further. Over hundreds of millions of years, assuming molecular binding is still possible, this susy world has the ingredients and the potential to build DNA and, in principle, reconstitute a susy counterpart to each previously existing carbon-oxygen based life form.

A similar reduction down to Oxygen will happen in stars but heavier elements will then be produced by fusion with no barrier at iron such as exists in the broken susy world. The lifetime of susy stars might, therefore, be expected to be many times longer than that of stars in the broken susy phase.

Without further input, we cannot say anything about the probable future lifetime of our current phase. The susy transition is an example of a decay of the false vacuum treated some decades ago by Coleman and collaborators [16]. This is a quantum tunneling event which could happen at any time even if the most probable time is arbitrarily far in the future. As a crude estimate of this time we can note that the probability per unit time per unit volume to nucleate a critically sized bubble and therefore affect a phase transition was given [16] in the form

$$
\frac{d P}{d t d V}=A e^{-B}
$$

where in a vacuum with non-zero energy density $\epsilon, B$ takes the form

$$
B(v a c)=\frac{27 \pi^{2} S^{4}}{2 \epsilon^{3}}
$$

Here $S$ is the surface tension of the susy bubble of true vacuum surrounded by the broken susy false vacuum . At any time, $\mathrm{t}$, at which the universe has volume, $\mathrm{V}(\mathrm{t})$, the probability per unit time to nucleate a critical bubble which will grow to engulf the universe is

$$
\frac{d P}{d t}=A V(t) e^{-B}
$$

In the presence of a vacuum energy density, $\epsilon$, the scale factor will satisfy

$$
\frac{\ddot{a}}{a}=-\frac{4 \pi G_{N}}{3}\left(\rho_{v a c}+3 p_{v a c}\right) .
$$

Putting $p_{v a c}=-\rho_{v a c}=-\epsilon$, this has the solution

$$
a(t)=e^{\gamma t / 3} a(0)\left(1+\left(\frac{3 a(0)}{\gamma a(0)}-1\right) \frac{1-e^{-2 \gamma t / 3}}{2}\right)
$$


where, in terms of Newton's constant, $G_{N}$,

$$
\gamma=\sqrt{24 \pi G_{N} \epsilon}
$$

Neglecting sub-leading terms, we may write the volume of the universe at time $t$ in terms of its present volume $V(0)$ as

$$
V(t)=V(0) e^{\gamma t}
$$

The natural time scale for the growth in volume of the universe is

$$
\gamma^{-1}=5.61 \cdot 10^{9} y r
$$

The volume of the universe is at least as big as the Hubble volume

$$
V(0)>V_{H}=7.79 \cdot 10^{78} \mathrm{~m}^{3} .
$$

The integrated probability that the universe will undergo the susy phase transition in a time $t$ from some starting time $t=0$ is, therefore,

$$
P(t)=\frac{A V(0)}{\gamma} e^{-B}\left(e^{\gamma t}-1\right) .
$$

$P(t)$ greater than unity implies multiple critical bubble formation [17] but even a single critical bubble will take over the universe. Therefore, we can use the time, $T$, at which $P(T)=1$ as an estimate of the probable time before the nucleation of a susy bubble destined to take over the universe.

$$
T=\gamma^{-1} \ln \left(1+\frac{\gamma}{A V(0)} e^{B}\right)
$$

Frampton [17] has argued, subject to some assumptions, that the critical radius should be at least of galactic size. This leads to a minimum value for the surface tension of $S=0.22$ $(\mathrm{MeV})^{3}$. The parameter $A$ is estimated [17, largely on dimensional grounds, to be of order $\epsilon$. These values are roughly consistent with those needed [12] to explain the gamma ray bursts as susy phase transitions in dense white dwarf stars. Unless the volume of the universe is much greater than the Hubble volume, they lead to a value of $T$ that is extremely large even compared to the large natural time scale of eq. 3.8 since, then, $B=1.5 \cdot 10^{99}$. This conclusion is not significantly changed by keeping the sub-leading terms in eq. 3.5.

The correct question to ask, however, is not the probability for a critical bubble to be formed somewhere in the universe, but instead what is the probability that such a bubble will strike Earth or some other location in a given time. Once nucleated somewhere in the universe, the bubble will require some time to propagate to any particular location such as that of Earth. If we take this local point at the present time as the space-time origin, the probability per unit time for a susy bubble to arrive at time $t$ is the probability per unit time for a critically sized bubble to be nucleated at any position $r^{\prime}$ at the retarded time $t^{\prime}=t-r^{\prime} / c$

$$
\frac{d P(0, t)}{d t}=\int d^{3} r^{\prime} e^{\gamma t^{\prime}} \frac{d P\left(r^{\prime}, t^{\prime}\right)}{d V^{\prime} d t^{\prime}} d t^{\prime} \delta\left(t^{\prime}-t+r^{\prime}\right)=e^{\gamma t} A e^{-B} \int d^{3} r^{\prime} e^{-\gamma r^{\prime}}
$$


This can be written

$$
\frac{d P(0, t)}{\gamma d t}=e^{\left(\gamma t-B+\ln \left(8 \pi A / \gamma^{4}\right)\right)}
$$

An integrated probability over any time interval exceeding unity should be interpreted as the probable number of susy bubbles hitting the earth in that time interval. Requiring that the integrated probability from the big bang to now $(t=0)$ be less than unity suggests

$$
B>\ln \left(8 \pi A / \gamma^{4}\right)
$$

or, using 3.2

$$
S>\left(\frac{2 \epsilon^{3}}{27 \pi^{2}} \ln \frac{8 \pi A}{\gamma^{4}}\right)^{1 / 4}=0.62 \cdot 10^{23}(\mathrm{~mm})^{-3}=4.91 \cdot 10^{-7}(\mathrm{MeV})^{3} .
$$

The numerical value here is based on the estimate of ref. [17 for $A$ although it is only weakly dependent on this estimate. On the other hand, this lower limit for $S$ is far below the estimated lower limit of ref. [17] discussed above. If we allow ourselves to consider saturating the limit 3.14, there is a non-negligible probability that the Earth will be swallowed by a susy bubble in a time $T$ from today that is smaller than $1 / \gamma$. This can be seen by integrating eq. 3.13 from 0 to $T$ :

$$
P(T)=\left(e^{\gamma T}-1\right) e^{\left(-B+\ln \left(8 \pi A / \gamma^{4}\right)\right)} .
$$

This is only relevant while $P(T)<1$ since the collision of multiple susy bubbles with Earth is superfluous.

The question, therefore, is whether such small values of $S$ as in eq. 3.15 are truly ruled out. Frampton's lower limit on $S$, cited above, relied on the assumption that the dark energy can exchange energy with that of galactic magnetic fields. Since no specific model was proposed in which this happens, one is still free to consider lower values of $S$ limited only by the current longevity of the universe as given in eq. 3.15. It remains to be seen whether such low values of $S$ are inconsistent with the susy phase transition being the central engine of gamma ray bursts [12]. Significantly larger values of $S$ imply that the broken susy universe will probably survive for a time large compared to $\gamma^{-1}$.

\section{Conclusions}

The present article opens the discussion of the consequences of a possible novel scenario for the end-phase of the universe. Our considerations are intended to be non-speculative except in so far as current theoretical ideas in the string landscape picture are speculative. Nevertheless, we can anticipate some reasonable criticisms of this work.

It could be argued that we have presented more detail than is justified by the uncertainties involved. On the other hand, there will be some who feel that dissemination of the ideas in 
the current paper should be discouraged until a greater depth of analysis has been achieved. We have, obviously, raised more questions than we have answered. The main results we would like to put before the physics community are those that follow in a straightforward way from the effective relaxation of the Pauli principle in a susy world. We have neither confirmed the possibility of life on this world nor conclusively ruled it out. When we return we will want a molecular physicist and a biochemist on board.

It will be objected by some that we have proposed the investigation of a world removed from the possibility of experimental confirmation and that our considerations are, therefore, outside the realm of physics. The same criticism has been directed at other string theory inspired suggestions that there are alternate worlds beyond the visible universe and outside of causal contact with us.

In fact, however, we are not ready to admit that there is no possibility of experimental confirmation of the existence of other worlds including the world of exact supersymmetry.

First of all, although perhaps a priori unlikely, it is not impossible that a solar system or even a small galaxy exists in a susy bubble within range of our telescopes. This would require that the critical radius in that environment be less than the actual radius of the physical system and that the actual radius be less than the critical radius in vacuum. In such a situation, the bubble would be prevented from expanding into the intergalactic space. This mechanism for confining a susy bubble has been discussed in [12. To briefly reiterate, the critical radius for a bubble in the vacuum is governed by the surface tension and the dark energy.

$$
R_{c}=\frac{3 S}{\epsilon}
$$

At nucleation or at any later stage in its development, a bubble will grow if its radius is greater than the critical radius and otherwise be quenched. The surface tension, $S$, is such that the critical radius in vacuo may be at least of the size of a typical galaxy [17. In a dense medium the denominator of eq. 4.1 will be replaced by the energy density advantage of a transition to true vacuum [12] and therefore the critical radius can be much smaller. A decreased density outside of a physical system can thus have the effect of preventing further growth of the bubble.

Since photons and other standard model particles which are light in both phases can easily pass through the susy domain wall, it might be possible to search for anomalous spectra from distant stars or galaxies. Also, any intelligent life existing in such a susy galaxy might be capable of sending radio waves into the broken susy world. Thus a susy civilization could, in principle, be found in the ongoing Search for Extraterrestrial Intelligence (SETI).

Secondly, there is nothing in principle which would prevent a space probe from entering such a susy bubble. Of course, such a probe would be immediately vaporized due to the collapse of its Pauli towers once it entered a susy region. Nevertheless, a final signal could be sent back to earth before crossing the domain wall. Since the susy world will be quite luminous, it should not be a problem to rule out a black hole as the cause of the probe's disappearance. 
If the effective potential is dynamically determined as in the string landscape and if the state of lowest vacuum energy density is supersymmetric, the supersymmetric world must eventually take over from the broken susy world. It is possible that the gamma ray bursts observed coming from distant galaxies are an advance sign of this coming transition although the expected time of the transition might be in the distant future.

\section{Acknowledgements}

This work was supported in part by the US Department of Energy under grant DE-FG0296ER-40967. We gratefully acknowledge discussions with Irina Perevalova on the subject of nuclear binding energies and with Zurab Berezhiani on the subject of phase transition theory.

\section{References}

[1] R. Bousso and J. Polchinski, JHEP 0006: 006 (2000)

L. Susskind, hep-th /0302219

[2] Ben Freivogel, M. Kleban, M.R. Martinez, L. Susskind, hep-th/0505232

[3] S. Kachru, R. Kallosh, A. Linde, and S. Trivedi, Phys. Rev. D68, 046005 (2003), hep-th/0301240

[4] Steven Giddings, hep-th/0303031, Phys. Rev. D68,026006 (2003)

[5] L. Jarv, T. Mohaupt, F. Saueressig, hep-th/0403063, JCAP, 0408:016 (2004)

Steven Giddings and Robert Myers, hep-th/0404220, Phys. Rev. D70, 046005 (2004)

[6] Review of Particle Physics, S. Eidelman et al, Phys. Lett. B592, 1 (2004)

[7] L. Clavelli, Phys. Rev. D45, 3276 (1992)

[8] S. Weinberg, Phys. Rev. Lett. 59, 2607 (1987)

[9] B. Povh et al Particles and Nuclei (an Introduction to the Physical Concepts), SpringerVerlag, New York (1995)

[10] T.E. Clark, S.T. Love, S.R. Nowling, hep-th/0108243 Nucl. Phys. B632,3 (2002)

[11] see for example C.H. Blanchard, C.R. Burnett, R.G. Stoner, R.L. Weber Introduction to Modern Physics, Prentice-Hall, New Jersey (1956)

[12] L. Clavelli and G. Karatheodoris, hep-ph/0403227, Phys. Rev. D72 ,035001 (2005); L. Clavelli and I. Perevalova, hep-ph/0409194, Phys. Rev. D71, 055001 (2005); L. Clavelli, hep-ph/0506215, Phys. Rev. D in press

[13] Huaiyu Duan, astro-ph/0401634; H.A. Bethe, Rev. Mod. Phys. 62, 801 (1990)

[14] F. Hoyle, D.N.F. Dunber, W.A. Wenzel, W. Whaling, Phys. Rev. 92, 1095 (1953) 
[15] R. Pichler, H. Oberhummer, A. Csoto, S.A. Moszkowski Nucl. Phys. A618, 55 (1997), nucl-th/9701028

H. Oberhummer, R. Pichler, A. Csoto, nucl-ph/9810057, Proceedings of Nuclei in the Cosmos V, ed. N. Prantzos (Editions Frontiers) (1999)

[16] S. Coleman, Phys. Rev. D15, 2929 (1977);C.G. Callan and S. Coleman, Phys. Rev. D16, 1762 (1977); S. Coleman and Frank DeLuccia, Phys. Rev. D21, 3305 (1980)

[17] P. Frampton, hep-th/0302007, Mod. Phys. Lett.A19, 801 (2004); hep-th/0508082 